\title{
Evaluación de Tecnologías Sanitarias: marco conceptual y perspectiva global
}

\author{
LUCY KUHN-BARRIENTOS ${ }^{1, a}$
}

\section{Health Technology Assessment: conceptual framework and international perspective}

The increasing concerns of nations to improve efficiency, access and quality of health care, have encouraged a more appropriate use of health interventions. Thus, the interest of clinicians and decision-makers has shifted towards evidencebased medicine, comparative effectiveness research and health technology assessment (HTA). Although these concepts should not be understood as synonyms, they converge on common characteristics: a systematic approach to evidence, the focus on relevant outcomes for the patient, and the notion that policy-making for a group of patients will affect others. As a consequence, concerns not only involve efficiency and effectiveness, but also transparency, clinical practice and opportunity costs. This paper introduces the concept and processes of HTA in the first and second sections. Section three reviews the evolution of HTA in developed and Latin-American countries, analyzing the aspects that influence the structure and scope of HTA. The last section concludes with reflections on the challenges to implement HTA in Chile.

(Rev Med Chile 2014; 142 (S1): S 11-15)

Key words: Chile; Decision making; Resource allocation; Technology assessment.

\section{¿Qué se entiende por evaluación de tecnologías sanitarias (ETESA)?}

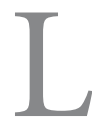

a ETESA es una disciplina que integra los aspectos clínicos, sociales, económicos, léticos y organizacionales que influyen en la incorporación de una tecnología. Los cuales son analizados siguiendo un proceso sistemático y riguroso de evaluación ${ }^{1}$. Su objetivo es proveer información de calidad a los tomadores de decisión, para formular políticas de salud seguras, costoefectivas y centradas en el paciente. Existen varias definiciones que enmarcan el concepto de ETESA, las cuales coinciden en que es una manera de evaluar la forma en que la ciencia y la tecnología son usadas en el cuidado de la salud y en la prevención de enfermedades ${ }^{2}$.

El Glosario de la International Network of Agencies for Health Technology Assessment (INA$H T A$ ) señala que es "un proceso sistemático de

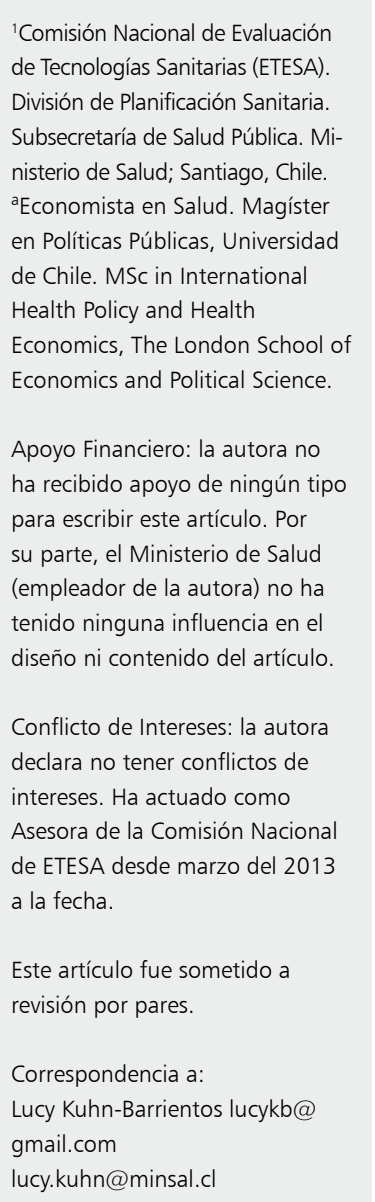

valoración de las propiedades, los efectos y/o los impactos de la tecnología sanitaria. Puede abordar tanto las consecuencias directas y deseadas, como las indirectas y no deseadas. Su objetivo principal es informar la toma de decisiones en atención sanitaria. La ETESA es realizada por grupos multidisciplinarios utilizando marcos analíticos explícitos basados en diversos métodos"

En este contexto, se entiende por tecnología sanitaria los métodos de diagnóstico y tratamiento, dispositivos médicos, medicamentos, vacunas, métodos de prevención y rehabilitación, así como también los sistemas organizacionales y de soporte usados para entregar las prestaciones de salud ${ }^{3}$.

\section{La ETESA y sus procesos}

La ETESA responde a un proceso dinámico y evolutivo. Abarca diferentes tipos de evaluacio- 
nes para informar las decisiones de asignación de recursos en cuanto al "valor" en términos de beneficios, riesgos y costos, de tecnologías nuevas y existentes ${ }^{4}$. En principio, explora todos los elementos de valor de una tecnología, no restringiéndose a aquellos que pueden ser demostrados en un ensayo clínico. Requiere considerar la integración de las intervenciones médicas en el cuidado de la salud. Por ello debe contemplar el contexto específico en el que la tecnología será usada, como las prácticas de atención, la estructura del sistema, o precios de la atención e insumos. Así como los factores sociales, las consideraciones éticas en la cobertura o las preferencias en la valoración del estado de salud de la población.

Otro aspecto importante es evaluar el balance (trade-off) entre riesgos y beneficios de largo plazo de las tecnologías, para asegurarse que los beneficios clínicos esperados superen los efectos adversos no intencionales ${ }^{4}$. Asimismo, la incorporación de los costos permite analizar si los beneficios netos de financiar una determinada tecnología, son mayores al costo de oportunidad del uso alternativo de dichos recursos. La pregunta a responder es si la tecnología vale la pena en términos de los recursos utilizados y los beneficios sanitarios y sociales generados. De esta forma en el trade-off de costos y beneficios del uso de una tecnología, también intervienen elementos valóricos de equidad, ética o protección social, que podrían determinar decisiones de cobertura más allá del análisis de los costos monetarios ${ }^{4}$.

De acuerdo a Sullivan et al. ${ }^{5}$, un proceso ETESA distingue al menos cinco actividades: (i) horizonte de evaluación; (ii) priorización y determinación del tópico a evaluar; (iii) búsqueda y análisis de la evidencia; (iv) appraisal o valoración y (v) financiamiento e implementación.

El horizonte de evaluación implica un análisis previo de las tecnologías a evaluar, para determinar potenciales requerimientos de evidencia o implicancias presupuestarias. Esta etapa considera también la identificación de actores de interés clave que podrían participar en la definición del alcance de los estudios. En segundo lugar, debe establecerse un sistema de priorización de las tecnologías nuevas o existentes a evaluar. Luego, se realiza la recolección, revisión sistemática y análisis de toda la información disponible sobre la tecnología. El análisis puede incorporar estudios de eficacia, seguridad, efectividad, evaluación económica, y consideraciones éticas, sociales y organizacionales, entre otras. El appraisal (o consideración del valor) de los resultados analizados, forma parte del proceso de toma de decisiones, en que se emite un juicio sobre el valor de la evidencia para elaborar una recomendación. Finalmente, se evalúan aspectos de financiamiento, implementación y monitoreo de la recomendación.

\section{La ETESA en el contexto internacional.}

\section{Orígenes en países desarrollados}

Los inicios de la ETESA radican en el sector público de EEUU en 1972 en la Office of Technology Assessment (OTA), con el fin de responder a una necesidad de los congresistas y legisladores de contar con suficiente información para tomar decisiones, considerando los posibles efectos sociales, económicos y legales que puede conllevar la incorporación de una nueva tecnología ${ }^{6}$. La OTA no evaluaba exclusivamente tecnologías sanitarias, sino también otras relacionadas con medio ambiente, energía, telecomunicaciones, agricultura, transporte y defensa. Si bien la oficina fue desarmada por falta de fondos en 1995, tuvo un enorme impacto en las políticas públicas a nivel mundial ${ }^{7}$.

A fines de los 70s ETESA se fue extendiendo en Europa, realizándose en 1979 la primera conferencia europea de ETESA en Estocolmo ${ }^{8}$. En 1980 se creó la agencia Nordic Evaluation of Medical Technology (NEMT) como un esfuerzo colaborativo entre Dinamarca, Finlandia, Noruega y Suecia. En 1982 surgió el Comité para la Evaluación y Difusión de Innovaciones Tecnológicas en los hospitales de París (CEDIT). En 1985 los Países Bajos formaron el Consejo de Salud ( $G e-$ zondheidsraad). El Consejo Sueco de ETESA ( $S B U$ ) se creó en 1987 y el 2002 se establece el Directorio de Beneficios Dentales y Farmacéuticos (TLV). En España, Catalunya fue pionera con su Programa de Evaluación de Tecnologías en 1988,9-11.

El desarrollo de ETESA se potenció en las décadas siguientes, estableciéndose en el Reino Unido el National Coordinating Centre for Health Technology Assessment (NCCHTA) en 1993; el National Institute for Clinical Excellence (NICE) en 1999; el Scottish Medicines Consortium (SMC) y el All Wales Medicines Strategy Group (AWMSG) en $2002^{8}$. 
Por su parte, Francia creó la Alta Autoridad de la Salud (HAS) en el 2000 y en Alemania surgieron el Comité Mixto Federal (G-BA) el 2000 y el Instituto para la Calidad y Eficiencia Clínica (IQWiG) el 2004.

\section{Aspectos de estructura y alcance de ETESA}

Los principales factores que han impulsado el diseño e implementación de modelos de ETESA, han sido la tendencia creciente del gasto en salud, las preocupaciones respecto de la eficiencia en la provisión de atenciones médicas y la racionalidad en el uso de alta tecnología ${ }^{11}$. Estos factores se relacionan o incluso derivan de las imperfecciones del mercado de la salud, en el cual las asimetrías de información incrementan la incertidumbre para tomar decisiones ${ }^{12}$. Ante este escenario, la información y la investigación se tornan en insumos indispensables para decidir sobre la incorporación de nuevas tecnologías y políticas en salud.

La experiencia internacional ha demostrado que los modelos y procesos de ETESA varían entre países, no existiendo un modelo único, dependiendo del contexto estructural, organizacional y administrativo de sus sistemas de salud ${ }^{13,14}$. En el caso de Europa, por ejemplo, desde la perspectiva administrativa y organizacional, es posible encontrar entidades ETESA a nivel nacional (HAS en Francia, IQWiG y G-BA en Alemania, NICE en Inglaterra, TLV y SBU en Suecia, o la Agencia de Evaluación de Tecnología Sanitaria en España); a nivel regional o local como en Suecia con los county councils, o en 6 regiones autónomas en España; y a nivel hospitalario como el CEDIT en Francia o algunas pocas entidades a nivel de atención primaria en España ${ }^{13,14}$.

Por su parte, al considerar los procesos de ETESA, se encuentran diversas experiencias de elaboración de las evaluaciones y alcances de la recomendación. En Alemania el IQWiG lleva a cabo una parte de la evaluación, pudiendo subcontratar algunas secciones y otorgar recomendaciones, mientras que otros actores toman la decisión final. En el caso de la G-BA de Alemania, el NICE de Inglaterra, o el AWSMG de Gales, principalmente subcontratan las evaluaciones y toman decisiones de cobertura. Por su parte, en el TLV sueco, el SMC escocés y también en el NICE para evaluaciones de tecnologías únicas, la industria lleva a cabo la ETESA y estas agencias chequean la calidad de la evidencia, los modelos y resultados ${ }^{13,14}$.

\section{Visión de ETESA en Latinoamérica}

Latinoamérica es una región con una gran diversidad étnica, cultural y socioeconómica. A diferencia de países europeos o Canadá, los sistemas de salud son altamente fragmentados, enfrentando problemas de eficiencia, equidad, acceso y protección financiera ${ }^{15}$. No obstante, se ha convertido en una región con creciente uso de ETESAs en la toma de decisiones, contando con apoyo de entidades internacionales como la Organización Panamericana de la Salud (OPS) y el Banco Interamericano del Desarrollo (BID) $)^{15}$.

Durante los años 80 las incipientes actividades de ETESA no provocaron grandes cambios en las políticas de salud de los países latinoamericanos. Sin embargo, desde mediados de los 90 ETESA tomó fuerza a la luz de las reformas al sector salud que surgieron en los países de la región y de la colaboración de países con experiencia como España y Canadá ${ }^{16}$.

En sus inicios, ETESA fue utilizada para dar forma a los paquetes de beneficios en Argentina, Uruguay y Chile. Por su parte, países como Brasil y México cuentan con sistemas formales que explícitamente requieren ETESAs para la incorporación de nuevas tecnologías ${ }^{15}$.

De esta forma, entre los diferentes tipos de instituciones ETESA en Latinoamérica podemos encontrar Unidades dependientes de los Ministerios de Salud como por ejemplo la Unidad Coordinadora de Evaluación y Ejecución de Tecnologías en Salud (UCEETS) en Argentina ${ }^{17}$; el Departamento de Ciencia y Tecnología (DECIT) de Brasil; o el programa ETESA dependiente del Departamento de Tecnología Médica en Uruguay ${ }^{15,18}$. Estos programas por lo general cumplen el rol de coordinar los esfuerzos de ETESA y producir información de calidad para la toma de decisiones.

Otras entidades corresponden a agencias regulatorias como la Agencia Nacional de Vigilancia Sanitaria (ANVISA), que regula el precio máximo de los medicamentos y registra y autoriza la comercialización de tecnologías sanitarias en base a criterios ETESA; o la Comisión Nacional de Incorporación de Tecnología (CONITEC) que recomienda al Ministerio qué tecnologías financiar en el sector público, ambas en Brasil ${ }^{19}$. En el caso de México, se requiere que la incorporación de nuevas tecnologías en el sector público se respalden en una ETESA ${ }^{15}$. El Consejo de Salubridad General (CSG) emite las regulaciones en 
salud basadas en ETESA y el Centro Nacional de Excelencia Tecnológica (CENETEC) es la oficina nacional de ETESA desde el 2004 ${ }^{14,15}$. Finalmente, en Colombia se ha creado recientemente el Instituto de Evaluación Tecnológica en Salud (IETS) que proveerá recomendaciones técnicas a la Comisión de Regulación en Salud (CRES) ${ }^{15}$.

Todo este desarrollo, junto a las colaboraciones del Mercado Común del Sur (MERCOSUR), el Pacto Andino y el apoyo de la OPS, impulsaron la creación de la Red de Evaluación de Tecnologías Sanitarias para las Américas: RedETSA, convocando a doce países de la región (Argentina, Bolivia, Brasil, Chile, Colombia, Costa Rica, Cuba, Ecuador, México, Paraguay, Perú y Uruguay). Su objetivo principal es promover el intercambio entre ellos para apoyar el proceso de toma decisiones en cuanto a la incorporación, diseminación y uso de tecnologías en salud, considerando el contexto de cada país ${ }^{20,21}$.

\section{El contexto chileno y la ETESA}

En Chile la toma de decisiones de asignación de recursos en salud, no se ha basado en un proceso ETESA formal. Sin embargo, desde los años 90 han existido diversos programas de generación de evidencia y de procesos de priorización para definir el financiamiento y la cobertura de tecnologías sanitarias.

En 1997 se creó una Unidad ETESA en el Ministerio de Salud. Su objetivo fue elaborar evaluaciones y reportes basados en evidencia, en concordancia con las prioridades determinadas por el Ministerio. Por su parte, la promulgación de la ley de Garantías Explícitas en Salud ${ }^{21}$, ha promovido procedimientos de priorización de las intervenciones a incorporar en sus paquetes de beneficios. Además, a nivel ministerial se han elaborado una serie de guías de práctica clínica y de metodologías de evaluación económica, así como estudios de costo-efectividad.

Con todo, los desafíos que quedan para alcanzar una institucionalidad formal para el proceso de toma de decisiones basado en ETESA, son enormes. La estructura mixta pública-privada del sistema de salud, que funciona con lógicas distintas de cobertura y asignación de recursos; la fragmentación y segmentación del sistema; la escasez de capacidades instaladas entre los inves- tigadores, académicos y profesionales de la salud; o la propia idiosincrasia en las dinámicas de toma de decisiones, son algunos de los factores que se deben enfrentar para avanzar hacia un modelo ETESA en el país.

Afortunadamente, el interés y reconocimiento de ETESA por parte de las autoridades sanitarias en Chile han ido en aumento en la última década. Ello, junto con la recomendación de OPS y el acuerdo de los países latinoamericanos de incorporar la ETESA en las políticas y sistemas de salud $^{22}$, han sentado las condiciones para que Chile comience a diseñar un modelo de ETESA. Esto surge a partir de los esfuerzos y definiciones de la Comisión Nacional de ETESA, constituida el 2012 por Resolución del MINSAL ${ }^{23}$. Tiene como objetivo principal proponer un modelo de institucionalización de ETESA en Chile, para evaluar, regular y gestionar las tecnologías sanitarias, de manera de fomentar la transparencia, eficiencia y equidad en la toma de decisiones de asignación de recursos en salud.

\section{Referencias}

1. Política de la Comisión Europea en HTA. Disponible en: [http://ec.europa.eu/health/technology_assessment/ policy/index_en.htm] [Consultado 25 julio 2013].

2. Stevens A, Milne R, Burls A. Health technology assessment: history and demand. J Public Health Med 2003; 25 (2): 98-101.

3. International Network of Agencies for Health Technology Assessment (INAHTA). Health Technology Assessment (HTA) Glossary. Compiled by Facey K. Edited by, Topfer LA, Chan L on behalf of the INAHTA. July 2006, first edition.

4. Drummond M, Schwartz S, Jonsson B, Luce B, Neumann $\mathrm{P}$, Siebert U, et al. Key principles for the improved conduct of health technology assessments for resource allocation decisions. Int J Technol Assess Health Care 2008; 24 (3): 244-58.

5. Sullivan S, Watkins J, Sweet B, Ramsey S. Health Technology Assessment in Health-Care Decisions in the United States. Value Health 2009; 12 (2): 39-44.

6. The OTA Legacy 1972 - 1995. Disponible en: [http:// www.princeton.edu/ ota] [Consultado 25 julio 2013].

7. O'Donnell J, Sissi V, Pashos C, Miller D, Smith M. Health technology assessment: lessons learned from around the world - an overview. Value Health 2009; 12 (2): 1098-2015.

8. Velasco M, Borlum F, Palmhoj C, Busse R. Health 
Technology Assessment and Health Policy-Making in Europe: Current status, challenges and potential. Observatory Studies Series No 14. WHO 2008, on behalf of the European Observatory on Health Systems and Policies.

9. Sampietro-Colom L. Once upon a time... The rise and use of HTA throughout the world. Michael Quarterly. The Norwegian Medical Society 2012; 9: 90-4.

10. Carlsson P, Jonsson E, Werkö L, Banta D. Health Technology Assessment in Sweden. Int J Technol Assess Health Care 2000; 16 (2): 560-75.

11. Sampietro-Colom L, Asua J, Briones E, Gol J. History of health technology assessment: Spain. Int J Technol Assess Health Care 2009; 25 (1): 163-73.

12. Haas-Wilson D. Arrow and the Information Market Failure in Health Care: The Changing Content and Sources of Health Care Information. J Health Polit Policy Law 2001; 26 (5): 1031-44.

13. Stafinski T, Menon D, Philippon D, McCabe C. (2011). Health technology funding decision-making processes around the world: the same, yet different. Pharmacoeconomics 2012; 29 (6): 475-95.

14. ISPOR Global Health Care Systems Road Map. Disponible en: [http://www.ispor.org/htaroadmaps/] [Consultado 26 agosto 2013].

15. Pichon-Riviere A, Silva Elias F, Rivero V, Vaca C. Early Awareness and Alert Activities in Latin America: current situation in four countries. Int J Technol Assess Health Care 2012; 28 (3): 315-20.

16. Pan American Health Organization. Developing health technology assessment in Latin America and the Caribbean. Washington, DC. PAHO 1998. ISBN 9275
073775.

17. Rubinstein A, Pichon-Riviere A, Augustovski F. Development and implementation of health technology assessment in Argentina: two steps forward and one step back. Int J Technol Assess Health Care 2009; 25 (1): 260-9.

18. Banta D. Health Technology Assessment in Latin America and the Caribbean. Int J Technol Assess Health Care 2009; 25 (1): 253-4.

19. Banta D, Almeida R. The development of health technology assessment in Brazil. Int J Technol Assess Health Care 2009; 25 (1): 255-9.

20. Lanzamiento de la Red de Evaluación de Tecnologías Sanitarias (REDETSA). Disponible en: [http://www. paho.org/col/index.php?option=com_content\&view $=$ ar ticle\&id=1180:lanzamiento-de-la-red-de-evaluacion-detecnologias-sanitarias-redetsa\&catid=767\&Itemid=554 [Consultado 15 Julio 2013].

21. Ministerio de Salud de Chile. Ley No 19.966. Establece un Régimen de Garantías en Salud. Publicada en el Diario Oficial en septiembre 2004. Ultima modificación, abril 2012.

22. Organización Panamericana de la Salud (OPS/OMS). Resolución CSP28.R9. Evaluación e Incorporación de Tecnologías Sanitarias en los Sistemas de Salud. 28 a Conferencia Sanitaria Panamericana. 64ª Sesión del Comité Regional. Washington, D.C., EE.UU. Septiembre, 2012.

23. Ministerio de Salud de Chile. Resolución Exenta No 108. Crea la Comisión Nacional de Evaluación de Tecnologías Sanitarias. 18 de diciembre, 2012. 